\title{
CT enterography of pediatric Crohn disease
}

\author{
Jonathan R. Dillman • Jeremy Adler • \\ Ellen M. Zimmermann • Peter J. Strouse
}

Received: 9 August 2009 /Revised: 10 October 2009 / Accepted: 30 October 2009 /Published online: 20 November 2009

(C) Springer-Verlag 2009

\begin{abstract}
CT enterography is an important tool in the noninvasive diagnosis and follow-up of pediatric Crohn disease. This imaging modality is particularly useful for assessing extent of disease (including both intestinal and extraintestinal manifestations), response to medical therapy, and disease-related complications. The purpose of this article is to provide a contemporary review of CT enterography technique as well as the spectrum of intestinal and extraintestinal findings in pediatric Crohn disease.
\end{abstract}

Keywords CT Crohn disease $\cdot$ Children $\cdot$ Bowel imaging

\section{Introduction}

Crohn disease, a form of chronic inflammatory bowel disease (IBD), is common in the pediatric population. The current worldwide prevalence of Crohn disease is approximately 130 per 100,000 individuals and its incidence is

J. R. Dillman $(\bowtie) \cdot$ P. J. Strouse

Department of Radiology, C.S. Mott Children's Hospital, University of Michigan Health System,

Section of Pediatric Radiology, 1500 E. Medical Center Drive,

Ann Arbor, MI 48109, USA

e-mail: jonadill@med.umich.edu

\section{J. Adler}

Department of Pediatrics and Communicable Diseases,

Division of Pediatric Gastroenterology,

University of Michigan Health System,

Ann Arbor, MI, USA

\section{E. M. Zimmermann}

Department of Internal Medicine, Division of Gastroenterology, University of Michigan Health System,

Ann Arbor, MI, USA rising [1]. The incidence of pediatric Crohn disease in the United States is 4.56 per 100,000 individuals [1]. Approximately $15-25 \%$ of individuals with IBD will present during childhood ( $<18$ years of age) [1]. Crohn disease is characterized by transmural bowel inflammation, skip lesions, and episodic flares of disease activity interspersed with periods of remission. Initial diagnosis, establishing disease extent, monitoring response to medical management, and identification of disease-related complications typically relies on a combination of clinical assessment, endoscopy (both optical and capsule), and radiologic evaluation.

Radiologic evaluation of Crohn disease can include the use of radiography, barium studies (upper gastrointestinal series, small bowel follow-through [SBFT] examination, contrast enema, and, less commonly, enteroclysis), computed tomography enterography (CTE), magnetic resonance enterography (MRE), and sonography. CTE and MRE allow for complete radiologic evaluation of both the intestinal and extraintestinal manifestations of abdominopelvic Crohn disease. Benefits of CTE when compared to MRE include better spatial resolution, fewer motion-related artefacts, increased availability, greater radiologist familiarity, lesser cost, and shorter examination time. Benefits of MRE when compared to CTE include better contrast resolution and a lack of ionizing radiation. As recent studies have demonstrated similar sensitivities for CTE and MRE for the detection of small bowel Crohn disease $[2,3]$, MRE is likely to become the primary modality for imaging of the small bowel in children. In certain children, however, CTE will still be indicated, such as individuals with pacemakers and other MRI-sensitive implanted devices.

A study by Jamieson et al. [4] in the pediatric population concluded that CT shows sensitivity for the evaluation of small bowel involvement in inflammatory bowel disease 
that is equal to or better than that of SBFT examination. Their study also indicated that most children preferred CT to barium studies [4]. A prospective study by Hara et al. [5] evaluated adults with CTE, capsule endoscopy, ileoscopy, and SBFT examination. They found that CTE, capsule endoscopy, and ileoscopy have a higher yield in depicting mild to moderate findings of Crohn disease than SBFT examination, and that CTE is better for detecting transmural and extraluminal abnormalities [5]. Additionally, they concluded that CTE can depict nonobstructive Crohn disease of the small bowel when conventional techniques, such as ileoscopy and SBFT examination, produce negative or inconclusive findings [5].

The purpose of this article is to present a contemporary review of CT enterography technique as well as the spectrum of intestinal and extraintestinal findings in pediatric Crohn disease.

\section{Pediatric CTE technique and radiation dose reduction}

Pediatric CTE technique involves several modifications to standard abdominopelvic CT imaging protocols. Prior to imaging, a low-density oral contrast material (most commonly VoLumen, a low-density barium sulfate oral contrast agent made by Bracco, Princeton, NJ) is typically administered to the child (using a weight-based algorithm at our institution) to maximize bowel luminal distention [6-9]. Low-density contrast materials also allow for assessment of bowel mucosal hyperenhancement, a finding that can be obscured by higher-density oral contrast agents [6]. Orally administered water can also be used as a low-density oral contrast agent in CTE, although it may result in less optimal small bowel distention. CT enteroclysis is a less often used approach to opacify the small bowel in children. This technique is more labor-intensive than CT enterography, requires nasojejunal intubation of the child, and typically uses higher-density contrast material. Brown et al. [10] documented that $\mathrm{CT}$ enteroclysis can be safe, feasible, and accurate in depicting small bowel pathology in children.

CTE almost always utilizes intravascular iodinated lowosmolality (or iso-osmolality) contrast material unless specifically contraindicated. We administer an intravenous dose of $2 \mathrm{ml} / \mathrm{kg}$ (maximum dose, $125 \mathrm{ml}$ ) using a power injector whenever possible. While we prefer to image during the portal venous phase (approximately $70 \mathrm{~s}$ after the initiation of contrast material injection), in part to allow for adequate evaluation of the solid organs, others have described enteric-phase imaging approximately 40-45 s after the initiation of contrast material injection [7, 9]. Vandenbroucke et al. [9] concluded that Crohn disease is adequately depicted on both the enteric and portal venous imaging phases. While some have advocated the use of anti-peristaltic agents, such as glucagon, prior to imaging to minimize bowel-related motion artefacts, we believe that this intervention is unnecessary given the rapid speed with which images are acquired using modern multi-detector CT scanners.

Images should be acquired using multi-detector $\mathrm{CT}$ with submillimeter collimation to obtain an isotropic data set that allows for the creation of a variety of two-dimensional (2D) multiplanar reformatted and three-dimensional (3-D) reconstructed images. From the source data, we reconstruct $2.5-\mathrm{mm}$ axial images with $1.25-\mathrm{mm}$ section overlap for primary review. The submillimeter axial source images are generally only reviewed as a problem-solving tool. Multiplanar reformatted images, particularly in the coronal plane, might assist in the detection of pathology [11]. We generate both coronal and sagittal reformatted imaging series with a 2-mm section thickness. Finally, we create subvolume maximum intensity projection (MIP) and front-cut volume-rendered (VR) 3-D imaging series in the coronal plane.

A variety of methods can be employed to minimize pediatric radiation exposure related to CTE. First, only a single imaging phase should be acquired in order to adhere to the ALARA (as low as reasonably achievable) principle. Second, tube current $(\mathrm{mA})$ should be minimized, as this imaging parameter is directly proportional to radiation dose. Tube current can be selected by using a weight-based protocol or tube current modulation (a form of automatic exposure control). Finally, the use of other nonionizing imaging modalities, such as MRE or sonography, should be strongly considered, particularly for follow-up imaging. The effective radiation dose related to CTE in a 10-year-old child is approximately $3.5 \mathrm{mSv}$, compared to approximately 1.8-2.2 $\mathrm{mSv}$ for SBFT (using, on average, $5.1 \mathrm{~min}$ of fluoroscopy time and 3.3 abdominal radiographs) [12]. The effective dose of SBFT examination can be extremely variable, however, depending on the fluoroscope utilized, fluoroscopy time and number of radiographs acquired can be greater than CTE. The mean $\mathrm{CTDI}_{\mathrm{vol}}$ (a measure of average absorbed radiation dose) for pediatric (ages 2 to 19 years) CTE at our institution is 4.9 mGy (when using a standard adult $32-\mathrm{cm}$ body reference phantom), with a range from 1.29 to $12.95 \mathrm{mGy}$.

\section{Bowel-related findings}

Crohn disease can affect any portion of the gastrointestinal (GI) tract from the mouth to the anus and most commonly involves the small bowel, particularly the terminal ileum. CTE specifically allows for noninvasive detailed evaluation of the GI tract from the level of the distal esophagus through the anus. 
A variety of small and large bowel (as well as gastric) findings can be observed at CTE in pediatric Crohn disease. The most common findings include abnormal bowel wall thickening and hyperenhancement (Figs. 1, 2, 3, 4) [13, 14]. These findings generally become more pronounced with increasing inflammation and disease activity. Multiple patterns of bowel wall enhancement might be observed, depending on the degree of mucosal, muscularis propria, and serosal hyperemia, inflammation, and fibrosis. Differential bowel wall enhancement might result in a striated appearance, sometimes referred to as mural stratification or the target sign (Figs. 1 and 2) [13]. More homogeneous bowel wall enhancement suggests the presence of fibrosis [13]. A recent study by Adler et al. [15], however, has called this supposition into question, demonstrating that the presence of fibrosis cannot be independently identified or distinguished from inflammation at CTE in children and adults.
A study by Booya et al. [7] concluded that the most sensitive visual marker of Crohn disease at CTE is mural hyperenhancement followed by mural thickening. Another study, by Baker et al. [8], was able to distinguish normal terminal ileum from terminal ileitis of active Crohn disease using a combination of mural attenuation and mural thickness. Alterations in bowel wall thickness and enhancement at CTE over time might be useful to monitor changes in disease activity in response to therapy [16].

Bowel (and gastric) luminal diameter changes might be noted in Crohn disease. Areas of abnormal luminal narrowing with proximal dilatation, or strictures, can be caused by either active inflammation or fibrosis. Strictures can result in partial or complete bowel obstruction (Fig. 5) or gastric outlet obstruction (Fig. 6). Findings that suggest bowel obstruction include proximal luminal dilatation, distal luminal decompression, and more proximal stasis of intraluminal bowel contents ("small bowel feces" sign)
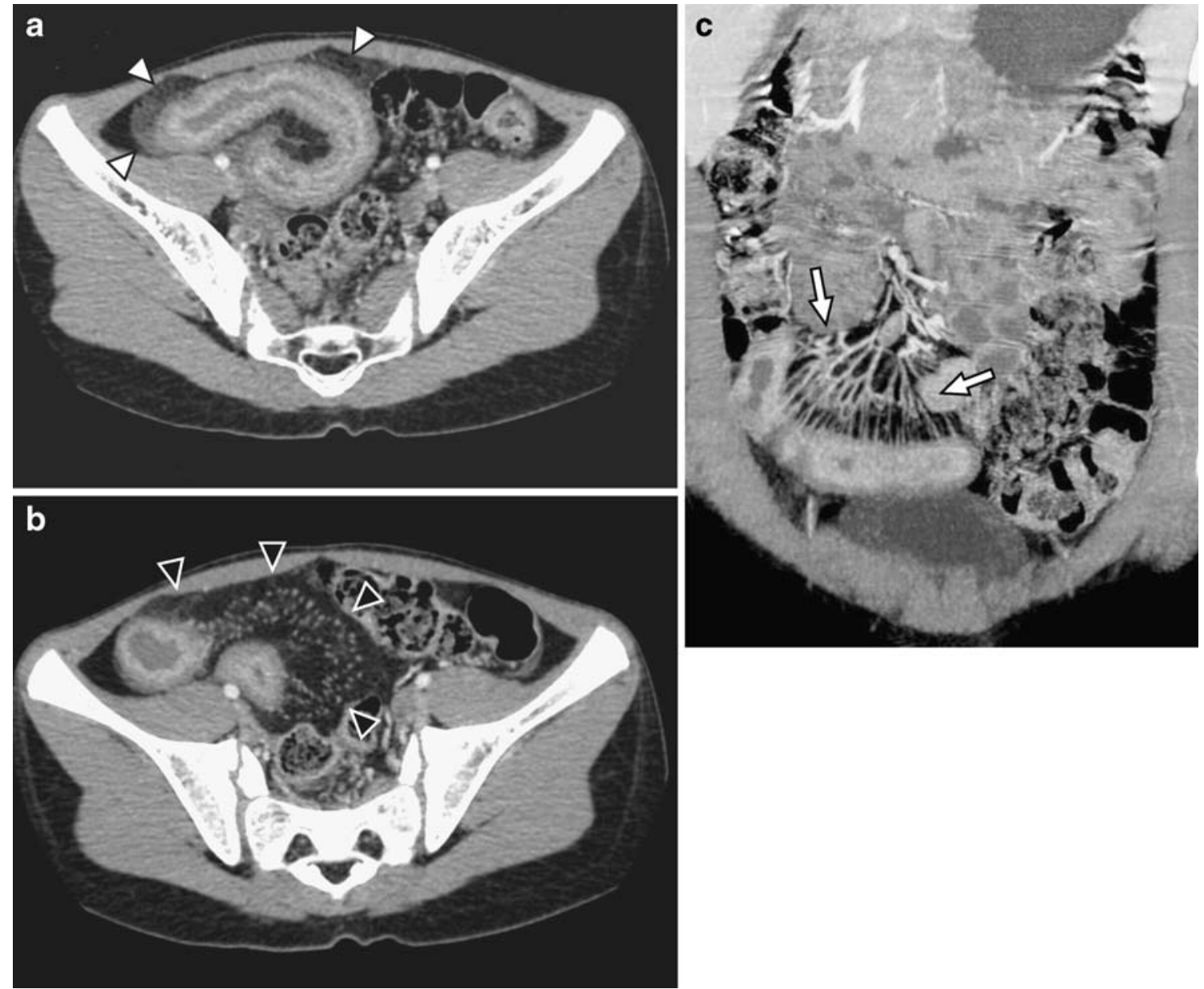

fibrofatty proliferation (black arrowheads). c Coronal-reformatted

Fig. 1 Crohn disease in an 11-year-old girl. a Axial CTE image reveals marked distal ileal mural thickening and differential mucosal and mural hyperenhancement (mural stratification). There is adjacent mesenteric fat inflammatory stranding (white arrowheads). b A slightly more caudal image reveals mesenteric hypervascularity and subvolume maximum-intensity projection image confirms the above findings. There is marked vasa recta prominence, the so-called comb sign (arrows) 

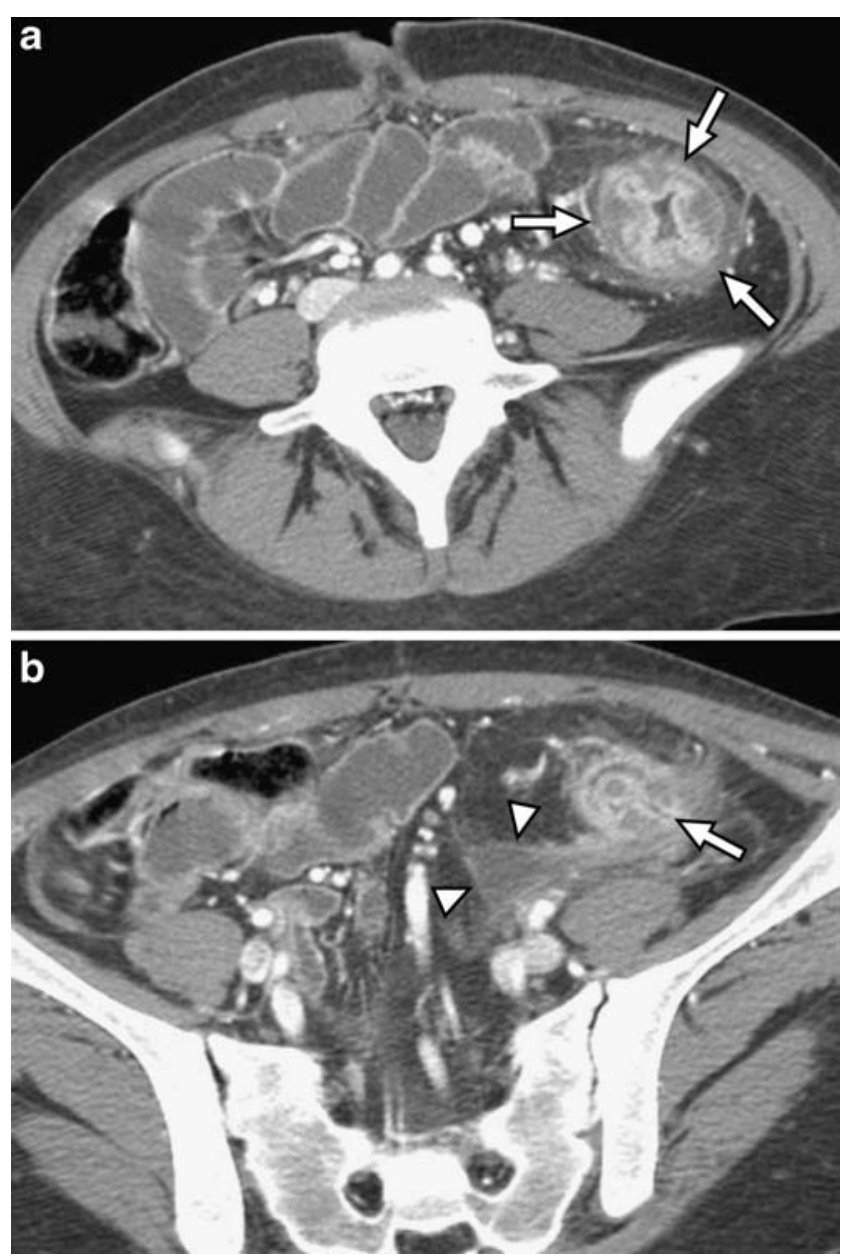

Fig. 2 Crohn disease in an 18-year-old with Down syndrome. a Axial CTE image demonstrates marked descending colon mural thickening (arrows) with adjacent fibrofatty proliferation, hypervascularity, and inflammatory fat stranding. Differential mucosal and serosal hyperenhancement result in mural stratification. b A more caudal image reveals an enhancing sinus tract (arrow) as well as an adjacent loculated fluid collection consistent with abscess (arrowheads)

(Fig. 7). There is no consensus on the value of CTE to distinguish between bowel obstruction caused by active inflammation versus fibrosis, and, therefore, the decision for surgical management should rely mainly on the response to medical management.

\section{Mesentery-related findings}

Several mesenteric abnormalities might be observed adjacent to bowel loops affected by Crohn disease. Mesenteric vasculature, particularly the vasa recta, can appear noticeably prominent because of hyperemia-related engorgement, sometimes referred to as the comb sign (Figs. 1, 3, and 4) $[13,17]$. A study by Lee et al. [17] found that perienteric and pericolic mesenteric hypervascularity correlated with the presence of active and more extensive Crohn disease.

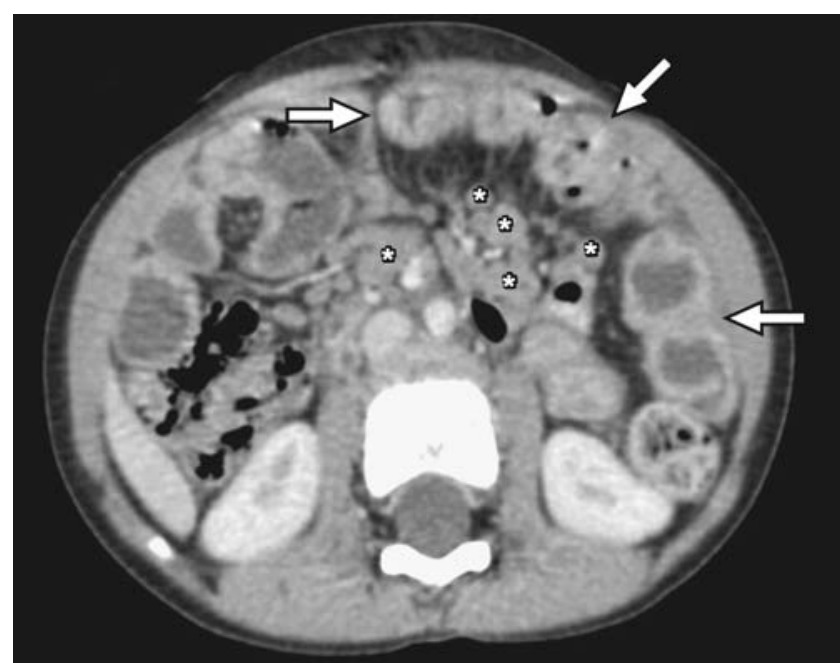

Fig. 3 Crohn disease in a 6-year-old girl. Axial CTE image reveals bowel wall thickening and hyperenhancement involving multiple loops of jejunum (arrows). Additionally, there is mesenteric lymph node enlargement (asterisks), inflammatory fat stranding, hypervascularity, and fibrofatty proliferation

They also found that increased mesenteric vascularity was associated with more common occurrence of longitudinal and perpendicular ulcerations at barium studies [17].

Mesenteric fat stranding, thought to be primarily caused by inflammation, is commonly observed in Crohn disease and can be variable in conspicuity (Figs. 1, 2, 3, 4, 7). Abnormal mesenteric fibrofatty proliferation, or "creeping fat," can also be seen adjacent to affected bowel loops (Figs. 1, 2, 3) [13, 14]. This finding might be particularly conspicuous in children who typically have a paucity of

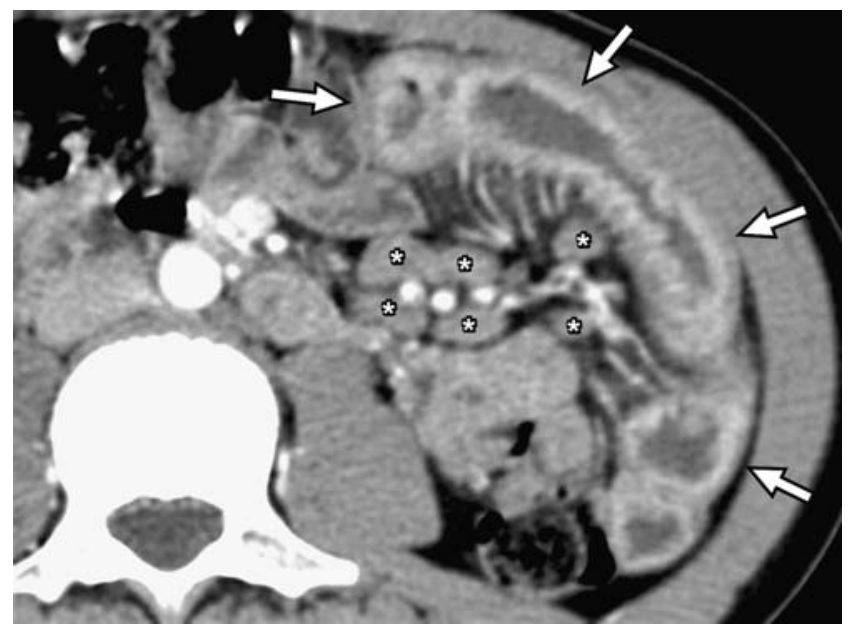

Fig. 4 Crohn disease in a 10-year-old boy. Axial CTE image through the left upper quadrant demonstrates jejunal mural thickening and both mucosal and mural hyperenhancement (arrows). A variety of mesenteric abnormalities are present, including increased number and size of lymph nodes (asterisks), hypervascularity, and inflammatory fat stranding 


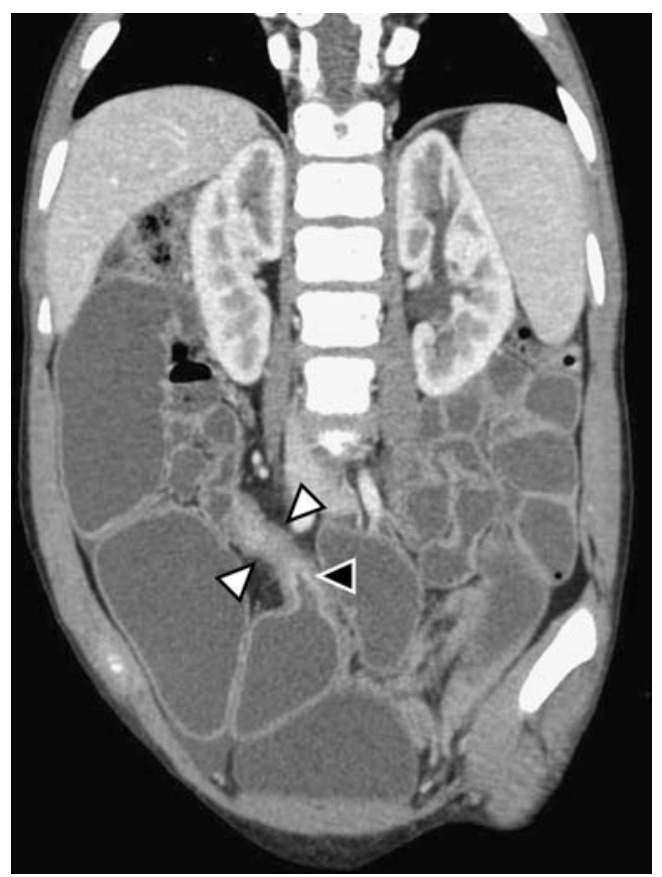

Fig. 5 Crohn disease in a 12-year-old boy. Coronal reformatted CTE image reveals a mid-ileal stricture (white arrowheads) within the right lower quadrant. A partial small bowel obstruction is present with abnormal dilatation of proximal small bowel loops, decompression of more distal small loops, and a discrete transition zone (black arrowhead)

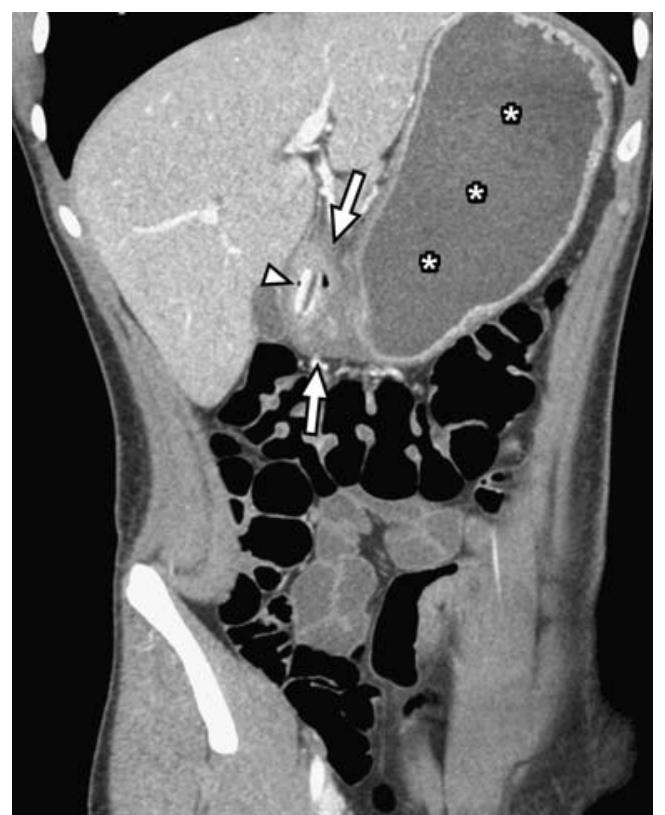

Fig. 6 Crohn disease in an 18-year-old man. Coronal-oblique reformatted CTE image demonstrates mural thickening of the distal stomach and proximal duodenum (arrows). There is marked gastric distention (asterisks) and diffuse gastric mucosal hyperenhancement. An orally ingested medication capsule (arrowhead) is lodged in the region of the pylorus with resultant gastric outlet obstruction

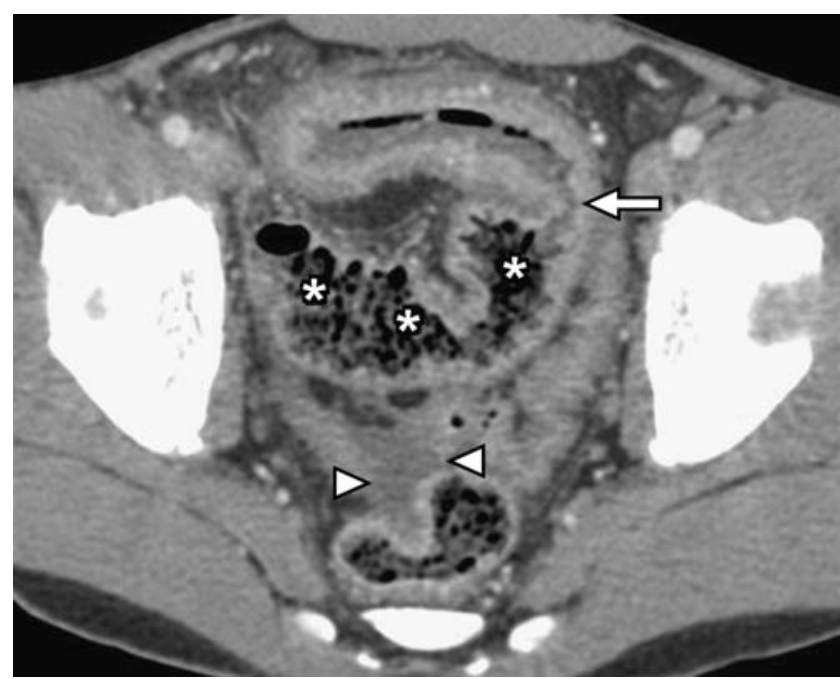

Fig. 7 Crohn disease in a 15-year-old boy. Axial CTE image through the distal ileum demonstrates mural thickening and both mucosal and mural hyperenhancement. There is a stricture (arrow) causing partial small bowel obstruction. More proximal ileum is dilated and appears to contain fecal debris, the "small bowel feces" sign (asterisks). Mesenteric inflammatory fat stranding is seen adjacent to abnormal ileum, and free fluid is present within the dependent portion of the pelvis (arrowheads). The sigmoid colon is decompressed within the left hemipelvis

mesenteric fat. Finally, mesenteric lymph nodes can be increased in number or size (Figs. 3, 4, and 8).

\section{Penetrating disease}

The bowel wall inflammatory changes of Crohn disease vary greatly in depth, with lesions being either superficial,

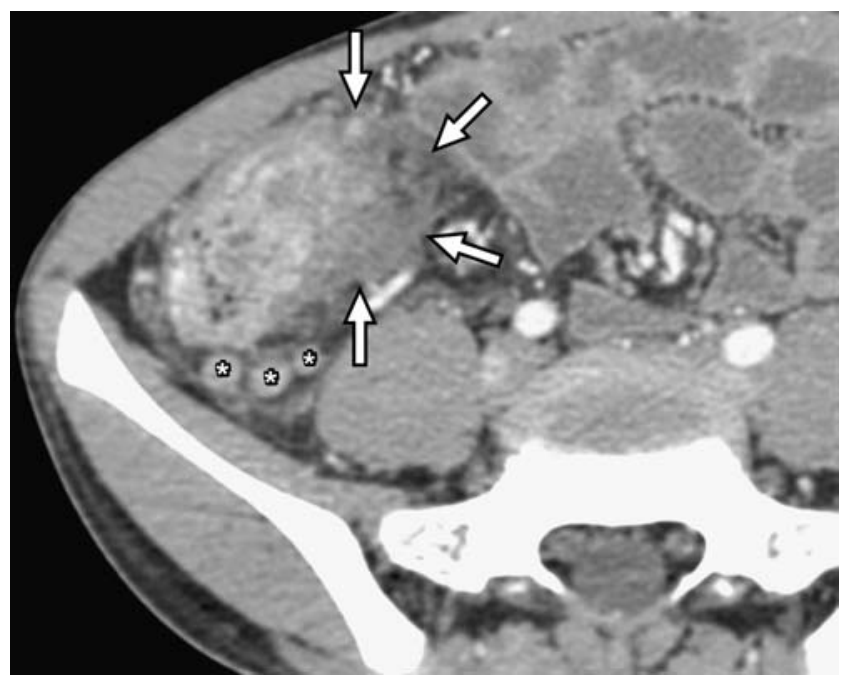

Fig. 8 Crohn disease in a 15-year-old boy. Axial CTE image through the right lower quadrant reveals marked abnormality of the ascending colon, including abnormal mural thickening and hyperenhancement. Adjacent complicated fluid and inflammatory fat stranding suggest the presence of phlegmon (arrows). Right lower quadrant lymph nodes are increased in size and number (asterisks) 
transmural, or extramural [13]. Superficial mucosal erosions and shallow (aphthous) ulcers are typically unable to be seen at CTE because of insufficient spatial resolution [13]. However, deep mural ulcers that extend into or through the muscularis propria are sometimes seen (Fig. 9) [13]. Deep mural ulcers present as focal bowel wall defects and can contain fluid or oral contrast material. Bowel wall hyperenhancement, likely related to the presence of inflammation and granulation tissue, might be seen immediately adjacent to the ulcer tract.

Ulcers that extend beyond the bowel wall result in sinus or fistula tract formation [13]. These tracts occur in approximately $20-40 \%$ of individuals with Crohn disease [13]. When such a penetrating tract is blind-ending and fails to communicate with a second epithelial surface or organ, a sinus tract is diagnosed. Sinus tracts commonly extend into the adjacent mesentery, although other structures, such as the body wall or psoas muscles, can be affected (Figs. 2 and 10). A fistula is diagnosed when a tract communicates with a second epithelial surface or organ. Fistulas in Crohn disease can involve numerous structures, such as adjacent bowel, stomach, bladder, uterus, fallopian tubes, vagina, scrotum, pancreas, and skin (Fig. 11) [13, 18].

Some form of perianal involvement in Crohn disease has been reported in $13-49 \%$ of pediatric patients $[19,20]$. Perianal lesions increase in prevalence with increasing duration of disease, eventually affecting up to $90 \%$ of individuals with Crohn disease [21]. Perianal fistulae and abscesses are usually symptomatic and may occur simultaneously (Fig. 12) [21]. Fistulae can be rectovaginal, intersphincteric, transphincteric, suprasphincteric, or extra-

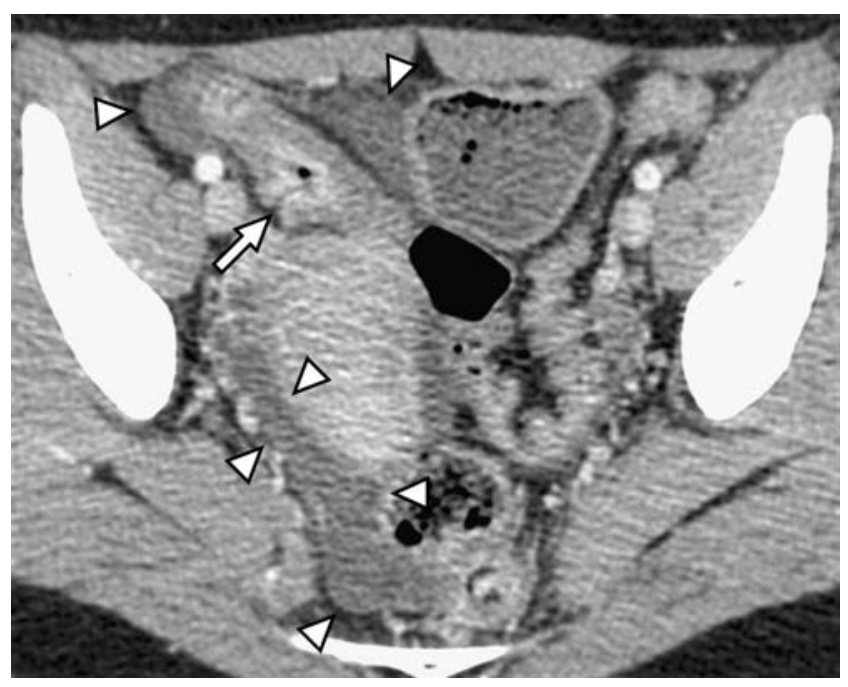

Fig. 9 Crohn disease in a 15-year-old girl. Axial CTE image demonstrates abnormal distal ileal wall thickening, mesenteric inflammatory fat stranding, and a moderate volume of intraperitoneal free fluid (arrowheads). A distal ileal deep mural ulcer (arrow) with hyperenhancing margins is present adjacent to the right external iliac vein sphincteric, while abscesses can be pararectal, ischiorectal, intersphincteric, perianal, or combined [21]. At CTE, pararectal, perianal, and ischiorectal inflammatory changes, including soft-tissue stranding and fluid, might be detected. Such changes sometimes extend to the skin surface. In recent years, MRI has emerged as the imaging modality of choice for the evaluation of perianal pathology, particularly fistulous disease [22].

\section{Intra-abdominal fluid}

Intraperitoneal free fluid is commonly observed in children with Crohn disease at CTE and is typically regarded as being reactive in etiology (Figs. 7 and 9). Complicated illdefined fluid (with HU often measuring greater than that of water) with associated mesenteric or omental inflammatory fat stranding suggests the presence of phlegmon [13] (Fig. 8). On occasion, areas of phlegmon develop into infected loculated rim-enhancing fluid collections, or abscesses (Fig. 2) [13, 14]. Such abscesses sometimes contain foci of gas [13].

\section{Other findings}

\section{Osseous}

Approximately $27 \%$ of all individuals with Crohn disease will have radiographic evidence of sacroiliitis, a manifestation of inflammatory bowel disease-related arthritis (that is commonly associated with HLA-B27 positivity) [23]. The prevalence of sacroiliitis can be even higher at CTE. When affected, the sacroiliac joints are generally symmetrically involved and might demonstrate subchondral sclerosis and articular surface erosions (Fig. 13). Changes of sacroiliitis at CTE can be very subtle in the pediatric population. Affected individuals might be asymptomatic [23] or experience only mild symptoms such as sacroiliac joint stiffness.

Osteopenia is common in children with Crohn disease, can lead to osteoporosis, and can increase risk for certain fractures [24]. The loss of bone mineral density is thought to be a result of a combination of poor nutrition (for example, vitamin D deficiency), circulating inflammatory cytokines that impair osteoblast function, and corticosteroid administration [1, 24]. Although subjective osteopenia might be observed at CTE when severe, dual-energy Xray absorptiometry (DEXA) is the traditional method by which bone mineral density can be quantified in patients with Crohn disease [1]. Peripheral quantitative computed tomography (pQCT) has also been recently shown to be useful in the assessment of bone mineral density in pediatric Crohn disease patients $[25,26]$. 


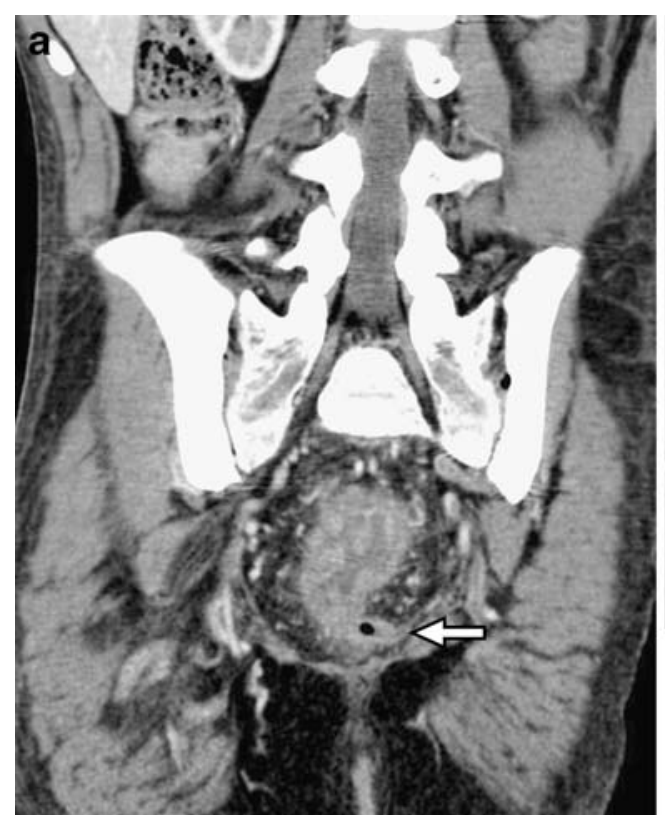

Fig. 10 Crohn disease in an 18-year-old man. a Coronal reformatted CTE image reveals rectal mural thickening, hyperenhancement, adjacent perirectal inflammatory fat stranding, and perirectal hypervascularity. A gas-containing sinus tract (arrow) arises from the left

\section{Urinary tract}

Crohn disease can affect the urinary tract in a variety of manners [16]. The prevalence of nephrolithiasis in Crohn disease patients compared to the general population is increased, with an incidence of 5-19\% [18]. Calcium

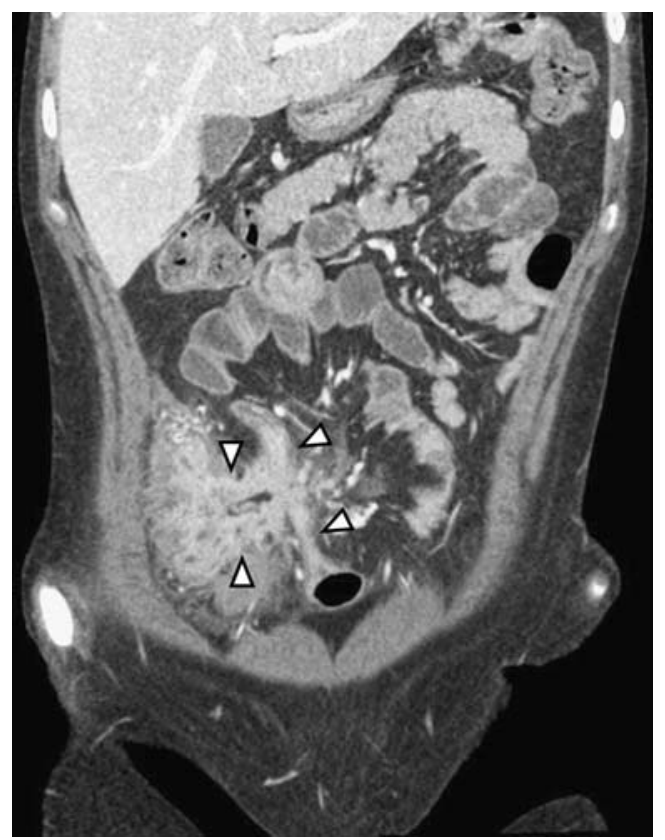

Fig. 11 Crohn disease in a 16-year-old girl. Coronal reformatted CTE image reveals multiple right lower quadrant enhancing fistula tracts (arrowheads) involving distal ileum, cecum, and ascending colon as well as adjacent mesenteric inflammatory fat stranding posterolateral aspect of the rectum. b Coronal reformatted CTE image through the kidneys reveals a nonobstructing calculus (arrow) within a left upper pole calyx

oxalate calculi commonly arise in the setting of terminal ileitis because of a relative increase in oxalate to calcium absorption from the GI tract (Fig. 10). Hydronephrosis in the setting of Crohn disease can have several causes, including urolithiasis and extrinsic ureteric compression by inflammatory changes or an abscess (Fig. 14) [18].

\section{Cholelithiasis}

Cholelithiasis is more common in individuals with Crohn disease than in the general population and is thought, at least in part, to be a result of terminal ileitis and decreased resorption of bile salts [27]. This interruption of enterohepatic circulation results in cholesterol-saturated bile and the formation of gallstones. While sonography is the preferred imaging modality for the evaluation of cholelithiasis, gallstones can appear as either hyperattenuating or hypoattenuating filling defects within the gallbladder lumen at CTE, depending on composition (Fig. 15). The sensitivity and specificity of thin-collimation multi-detector CT for the detection of cholelithiasis in vivo in children is unknown. The prevalence of cholelithiasis in Crohn disease patients increases with age [27].

\section{Conclusion}

A wide variety of CTE findings can be observed in pediatric Crohn disease, the majority similar to those observed in adults. Based on current practice patterns, 

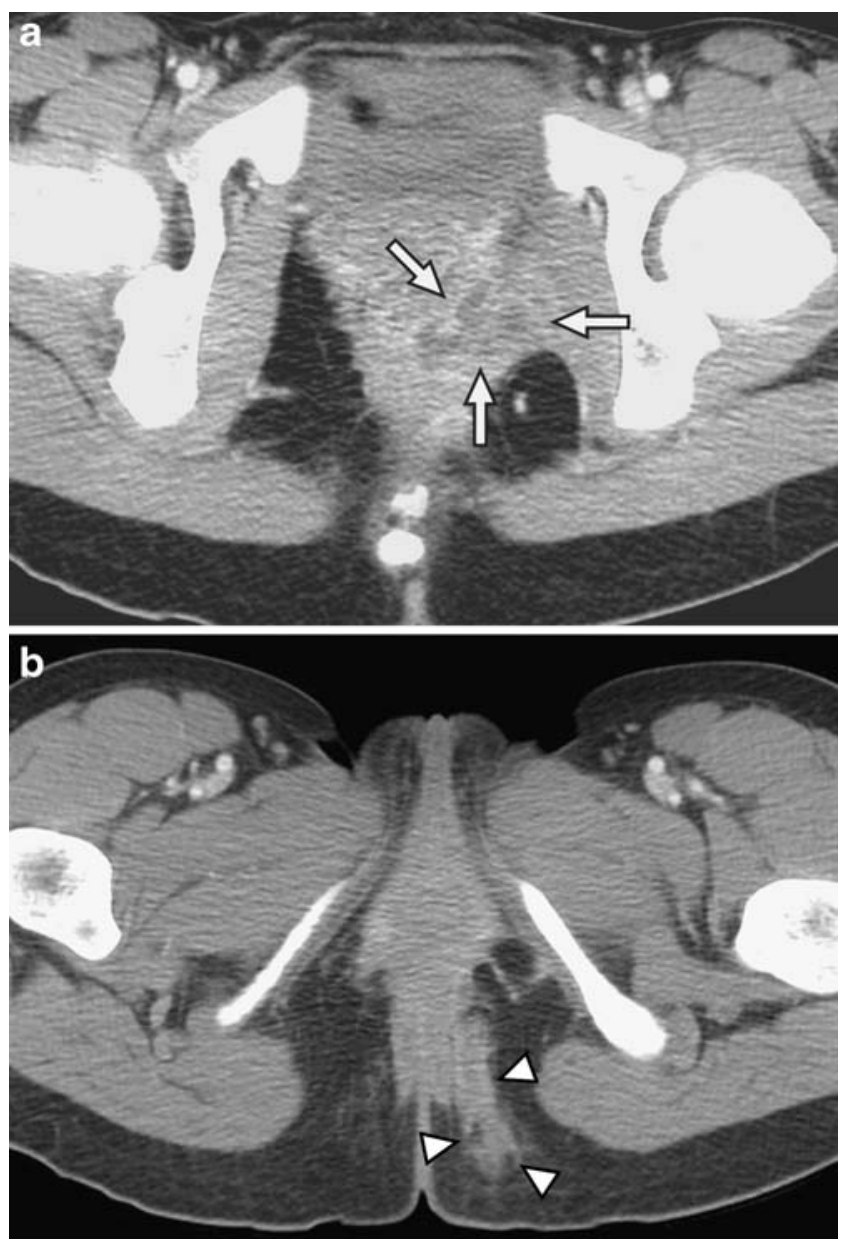

Fig. 12 Crohn disease in a 19-year-old woman. a Axial CTE image through the levator ani musculature reveals a low-attenuation focal fluid collection with adjacent hyperenhancement and inflammatory changes (arrows). Findings are consistent with abscess. b Another image through the level of the external anal sphincter reveals a fluidfilled left perianal fistula tract (arrowheads)

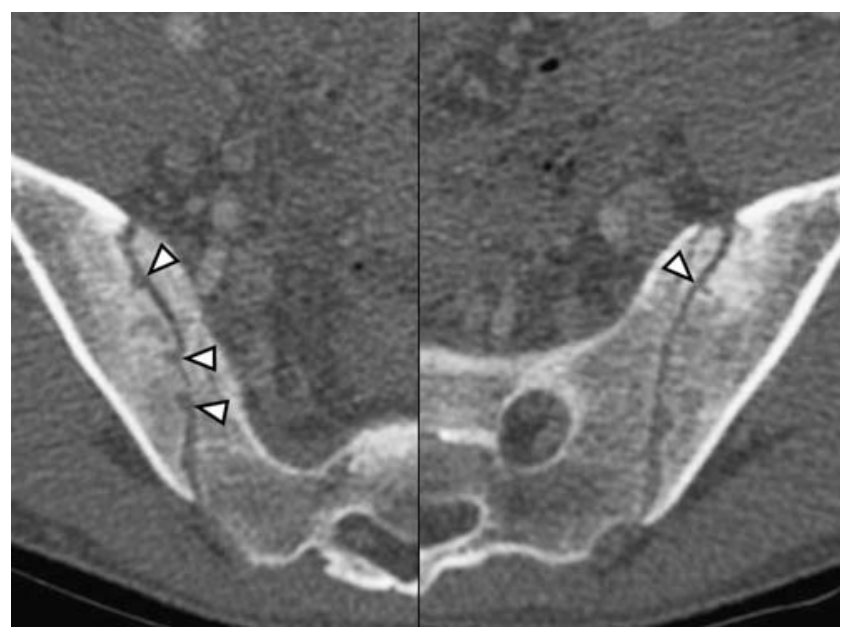

Fig. 13 Crohn disease in a 17-year-old boy. Axial composite CTE image of the right and left sacroiliac joints using a wide window/level setting reveals multiple tiny articular surface erosions (arrowheads) and subtle subchondral osseous sclerosis, consistent with symmetric sacroiliitis

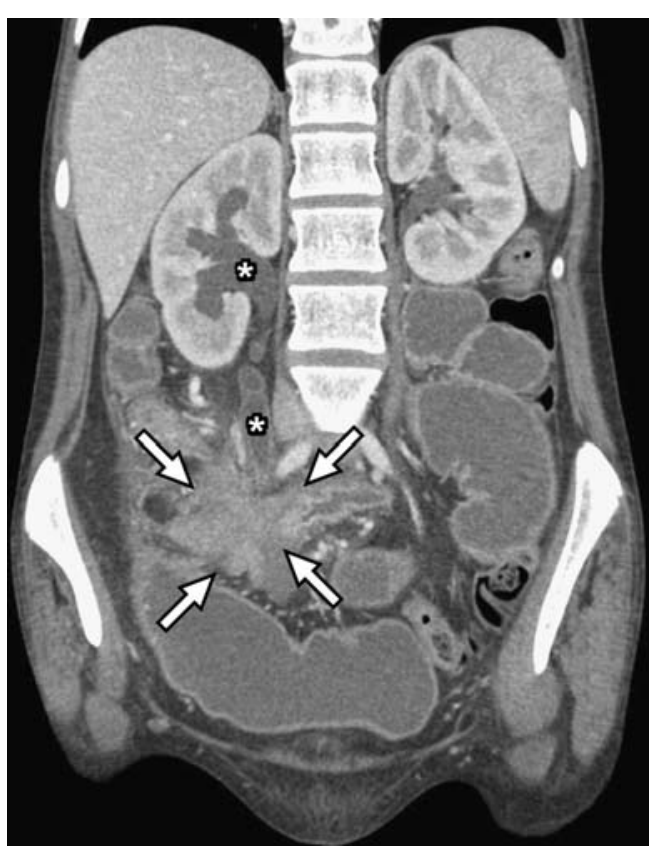

Fig. 14 Crohn disease and right hydronephrosis in a 16-year-old girl. Coronal reformatted CTE image demonstrates moderate right pelvicaliectasis and proximal ureterectasis (asterisks) caused by marked right lower quadrant inflammatory changes (arrows), including multiple enteroenteric fistulae and inflammatory small bowel stricturing with resultant partial bowel obstruction

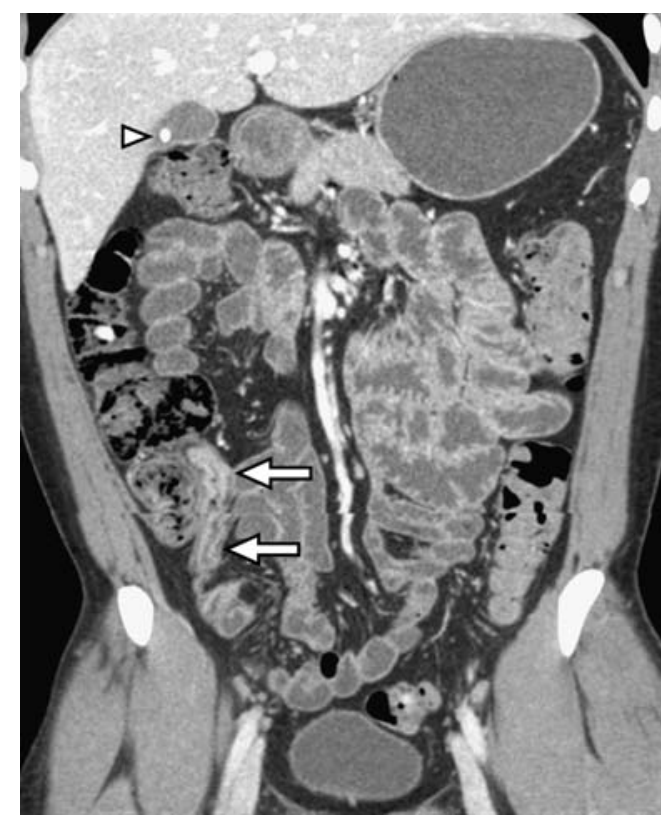

Fig. 15 Crohn disease in an 18-year-old man. Coronal reformatted CTE image reveals distal ileal mural thickening and mucosal hyperenhancement (arrows) as well as adjacent right lower quadrant mesenteric hypervascularity. A high-attenuation focus within the gallbladder lumen represents a gallstone (arrowhead) 
CTE plays an important role in the diagnosis of Crohn disease in children as well as in establishing disease extent and identifying disease-related complications. A variety of techniques minimize pediatric radiation exposure related to CTE. While CTE allows for complete assessment of intestinal and extraintestinal pediatric Crohn disease, it is our desire that MRE will soon become the primary imaging modality by which inflammatory bowel disease in children is assessed.

\section{References}

1. Kim SC, Ferry GD (2004) Inflammatory bowel diseases in pediatric and adolescent patients: clinical, therapeutic, and psychosocial considerations. Gastroenterology 126:1550-1560

2. Siddiki HA, Fidler JL, Fletcher JG et al (2009) Prospective comparison of state-of-the-art MR enterography and CT enterography in small-bowel Crohn's disease. AJR 193:113-121

3. Lee SS, Kim AY, Yang SK et al (2009) Crohn disease of the small bowel: comparison of CT enterography, MR enterography, and small-bowel follow-through as diagnostic techniques. Radiology 251:751-761

4. Jamieson DH, Shipman PJ, Israel DM et al (2003) Comparison of multidetector CT and barium studies of the small bowel: inflammatory bowel disease in children. AJR 180:1211-1216

5. Hara AK, Leighton JA, Heigh RI et al (2006) Crohn disease of the small bowel: preliminary comparison among CT enterography, capsule endoscopy, small-bowel follow-through, and ileoscopy. Radiology 238:128-134

6. Applegate KE, Maglinte DD (2008) Imaging of the bowel in children: new imaging techniques. Pediatr Radiol 38(Suppl 2): S272-S274

7. Booya F, Fletcher JG, Huprich JE et al (2006) Active Crohn disease: CT findings and interobserver agreement for enteric phase CT enterography. Radiology 241:787-795

8. Baker ME, Walter J, Obuchowski NA et al (2009) Mural attenuation in normal small bowel and active inflammatory Crohn's disease on CT enterography: location, absolute attenuation, relative attenuation, and the effect of wall thickness. AJR 192:417-423

9. Vandenbroucke F, Mortelé KJ, Tatli S et al (2007) Noninvasive multidetector computed tomography enterography in patients with small-bowel Crohn's disease: is a 40-second delay better than 70 seconds? Acta Radiol 23:1-9 [Epub ahead of print]
10. Brown S, Applegate KE, Sandrasegaran K et al (2008) Fluoroscopic and CT enteroclysis in children: initial experience, technical feasibility, and utility. Pediatr Radiol 38:497-510

11. Raptopoulos V, Schwartz RK, McNicholas MM et al (1997) Multiplanar helical CT enterography in patients with Crohn's disease. AJR 169:1545-1550

12. Gaca AM, Jaffe TA, Delaney S et al (2008) Radiation doses from small-bowel follow-through and abdomen/pelvis MDCT in pediatric Crohn disease. Pediatr Radiol 38:285-291

13. Toma P, Granata C, Magnano G et al (2007) CT and MRI of paediatric Crohn disease. Pediatr Radiol 37:1083-1092

14. Jabra AA, Fishman EK, Taylor GA (1994) CT findings in inflammatory bowel disease in children. AJR 162:975-979

15. Adler J, Punglia D, Dillman JR et al (2008) CT enterography findings correlate with tissue inflammation but not fibrosis in resected small bowel Crohn's disease. Gastroenterology 134:A195

16. Hara AK, Alam S, Heigh RI et al (2008) Using CT enterography to monitor Crohn's disease activity: a preliminary study. AJR 190:1512-1516

17. Lee SS, Ha HK, Yang SK et al (2002) CT of prominent pericolic or perienteric vasculature in patients with Crohn's disease: correlation with clinical disease activity and findings on barium studies. AJR 179:1029-1036

18. Simoneaux SF, Patrick LE (1997) Genitourinary complications of Crohn's disease in pediatric patients. AJR 169:197-199

19. Markowitz J, Daum F, Aiges H et al (1984) Perianal disease in children and adolescents with Crohn's disease. Gastroenterology $86: 829-833$

20. Tolia V (1996) Perianal Crohn's disease in children and adolescents. Am J Gastroenterol 91:922-926

21. Solomon MJ (1996) Fistulae and abscesses in symptomatic perianal Crohn's disease. Int J Colorectal Dis 11:222-226

22. Halligan S, Stoker J (2006) Imaging of fistula in ano. Radiology 239:18-33

23. Peeters H, Vander Cruyssen B, Mielants H et al (2008) Clinical and genetic factors associated with sacroiliitis in Crohn's disease. J Gastroenterol Hepatol 23:132-137

24. Leonard MB (2007) Glucocorticoid-induced osteoporosis in children: impact of the underlying disease. Pediatrics 119(Suppl 2):S166-S174

25. Dubner SE, Shults J, Baldassano RN et al (2009) Longitudinal assessment of bone density and structure in an incident cohort of children with Crohn's disease. Gastroenterology 136:123-130

26. Gilsanz V, Perez FJ, Campbell PP et al (2009) Quantitative CT reference values for vertebral trabecular bone density in children and young adults. Radiology 250:222-227

27. Parente F, Pastore L, Bargiggia S et al (2007) Incidence and risk factors for gallstones in patients with inflammatory bowel disease: a large case-control study. Hepatology 45:1267-1274 